\title{
Psoriasis with verrucous appearance: A case report
}

\section{Sarra Ben Rejeb', Amen Dhaoui', Dorra Ben Ghachem¹, Asmahane Souissi², Khadija Bellil ${ }^{1}$}

${ }^{1}$ Department of Pathology, FSI Hospital, La Marsa, Tunisia, ${ }^{2}$ Department of Dermatology, FSI Hospital, La Marsa, Tunisia

Corresponding author: Dr. Sarra Ben Rejeb, E-mail: sarrabenrejeb88@yahoo.fr

\begin{abstract}
Psoriasis verrucosa is a rare clinical variant of psoriasis with peculiar histologic features. Only few cases have been reported in the literature. We herein report a rare case of psoriasis with verrucous appearance occurring in a 63 year-old woman who presented with verrucous and scaly erythematous plaque of the legs which was developed thirty years ago. The biopsy specimen showed regular psoriasiform epidermal hyperplasia with acanthosis, hyperkeratosis, and focal spongiosis with a superficial perivascular infiltrate. The patient was diagnosed with verrucous psoriasis. Recognition of this entity should preempt confusion with verruca vulgaris or other entities capable of producing wart-like epidermal changes.
\end{abstract}

Key words: Psoriasis; Verrucous; Histology

\section{INTRODUCTION}

Psoriasis is an inflammatory disorder characterized by peculiar skin and joint manifestations. The most common clinical presentation is a scaly erythematous plaque with thick silvery white scale. Psoriasis with verrucous appearance is a rare variant of the disease with characteristic clinical and pathological features; it might be confused with many other lesions including warts, epidermal nevus, contact dermatitis, eczema and fungal infection $[1,2]$.

We herein describe another case of this rare entity. The clinicopathological features of this disease are discussed.

\section{CASE REPORT}

A 63 year-old woman with no past medical history presented with erythematous, scaly lesions of the lowers extremities developed thirty years ago. They were painless but were progressively growing, causing significant physical dis-figurement and discomfort on wearing shoes. Physical examination revealed verrucous plaques of the legs (Fig. 1). A skin biopsy of these lesions has been performed.
Pathologic examination revealed psoriasiform epidermal hyperplasia of rete ridges with prominent papillomatosis, marked parakeratosis and hyperkeratosis. It also showed epidermal hypogranulosis, thin suprapapillary epidermal plates, focal spongiosis of the Malpighi mucous body and a superficial perivascular inflammatory infiltrate. No inflammatory collection has been noted within the epidermal layers (Figs. 2 and 3). Koilocytic changes were not observed.

Clinical and pathological findings were consistent with verrucous psoriasis (VP). The patient was treated with local dermocorticoid. The patient has not been seen since the beginning of the treatment.

Prior to the study, patient gave written consent to the examination and biopsy after having been informed about the procedure.

\section{DISCUSSION}

Verrucous psoriasis is a rare variant of the disease with less than thirty cases reported in the literature [3]. It is mostly occurring on men with a sex ratio of 1.6 and a mean age of 53 year old $[1,4]$. Clinically, VP is characterized by a scaly erythematous plaque with

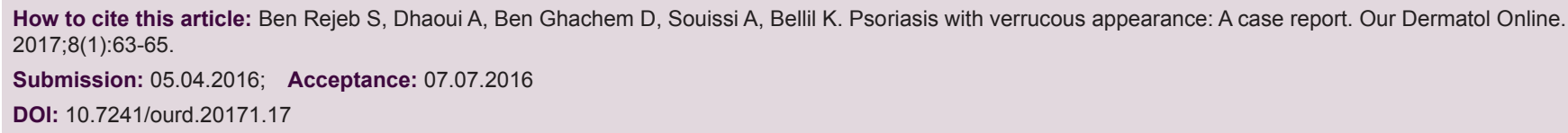




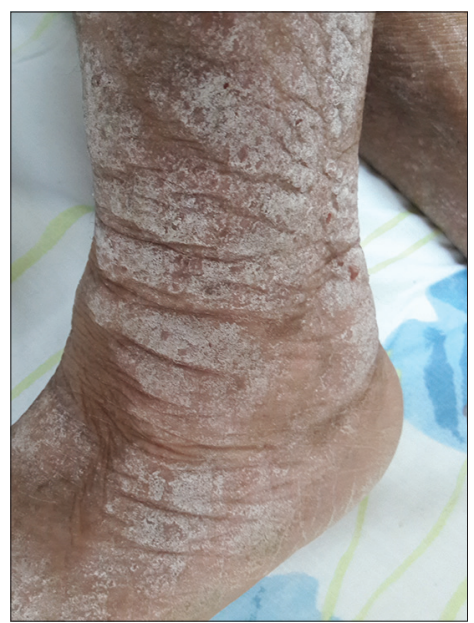

Figure 1: Warty plaques of the foot.

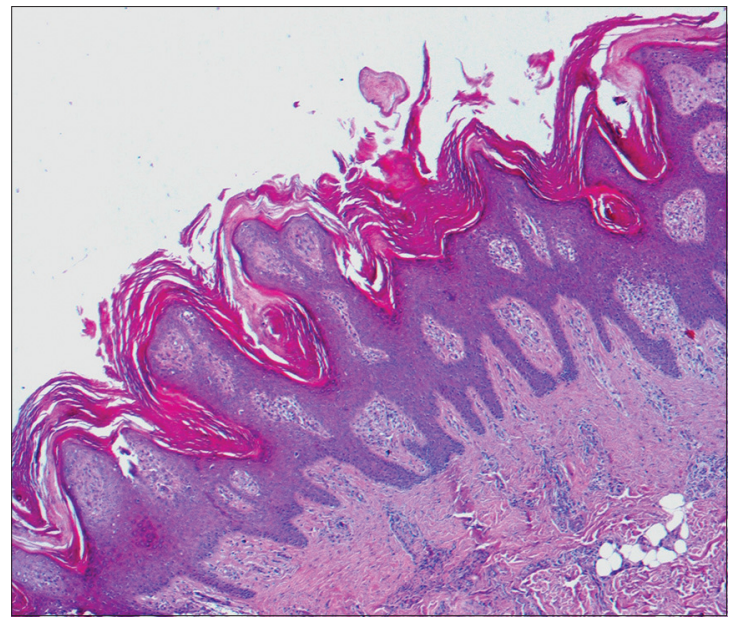

Figure 2: Wart-like appearance of the epidermis (HEx400).

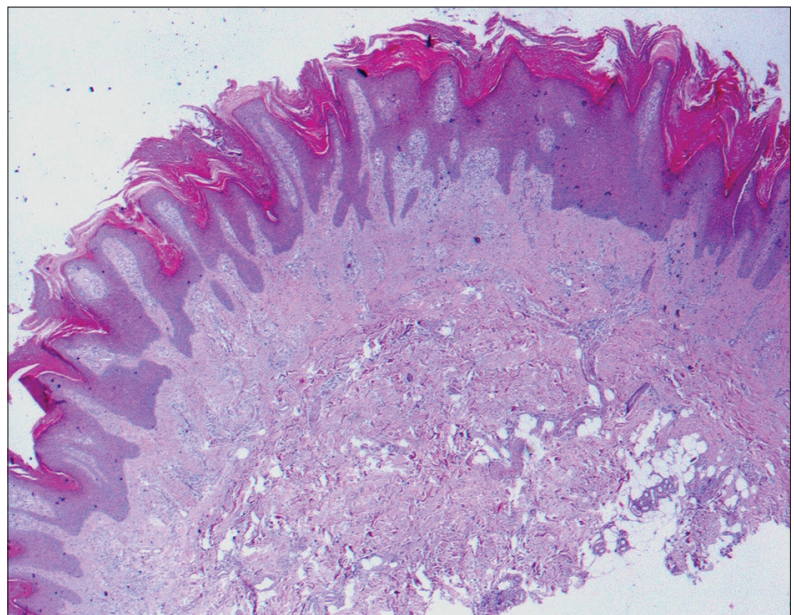

Figure 3: Epidermal hyperplasia with parakeratosis and prominent papillomatosis (HE x200).

wart-like changes. It is commonly occurring on frictions area such as elbows, hands, knees and feet. VP may be confused with many other benign lesions, including verruca vulgaris, epidermal nevus, contact dermatitis, eczema and fungal infection [1]. However, a verrucous carcinoma must be ruled out on biopsy. Microscopically, the lesion has a characteristic feature of psoriasis with wart-changes, it is typically showing parakeratosis, epidermal psoriasiform hyperplasia with elongation of rete ridges, thin suprapapillary epidermal plates, thinning of the granular layer, and dilated, tortuous capillaries with an inflammatory infiltrate, which may contain admixed neutrophils in the papillary dermis. Munro abscesses and spongiform pustules are frequently noted. In our case, despite the absence of characteristic neutrophils collection's of psoriasis within the epidermal layer, the correlation of clinical and other histologic features including papillomatosis and epithelial buttressing suggested the diagnosis. Moreover, Munro abscesses might be absent in 35\% of authentic psoriasis. Classically, absence of koilocytic changes and Human papilloma virus (HPV) immunostaining support the diagnosis of verrucous psoriasis.

The etiology of verrucous psoriasis remains unclear. It has been associated in some cases to lymphatic obstruction, microangiopathy, diabetes and obesity $[5,6]$. Other authors consider it as a progressive form of vulgaris psoriasis $[1,5]$. The verrucous changes in this case have been related to repeated trauma.

However, although it is considered to be a variant from psoriasis, little is known about its adequate treatment because of poor response to classic local therapy (dermocorticosteroid, vitamine $\mathrm{D}$, puvatherapy). Some authors reported the efficacy of etretinate, adalimumab and methotrexate. However, further investigations are required to determine an optimal treatment for this rare entity.

\section{CONCLUSION}

Verrucous psoriasis is a rare variant of psoriasis that might prompt consideration of verruca vulgaris. Combination of clinical and pathological findings is required for precise diagnosis. Because of its rarity, no codified therapy has been established yet.

\section{Consent}

The examination of the patient was conducted according to the Declaration of Helsinki principles. Written informed consent was obtained from the patient for publication of this article. 


\section{www.odermatol.com}

\section{REFERENCES}

1. Khalil FK, Keehn CA, Saeed S, Morgan MB. Verrucous psoriasis: a distinctive clinicopathologic variant of psoriasis. Am J Dermatopathol. 2005;27:204-7.

2. Monroe HR, Hillman JD, Chiu MW. A case of verrucous psoriasis. Dermatol Online J. 2011;17:10.

3. Kawtar I, Mariame M, Siham L, Fatimazahra M, Hassania A, Taoufiq H. Verrucous Psoriasis and Verrucous Lichen Associated With an Autoimmune Hepatitis. J Clin Diagn Res. 2014;2:2.

4. Curtis AR, Yosipovitch G. Erythrodermic verrucous psoriasis. J Dermatolog Treat. 2012;23:215-8.

5. Okuyama R, Tagami H. Psoriasis verrucosa in an obese Japanese man; A prompt clinical response observed with oral etretinate. J Europ Acad Dermatol Venereol. 2006;20:1359-61.

6. Zouboulis CC, Biczó S, Gollnick H, Reupke HJ, Rinck G, Szabó M, et al. Elephantiasis nostras verrucosa: beneficial effect of oral etretinate therapy. Br J Dermatol. 1992;127:411-6.

Copyright by Sarra Ben Rejeb, et al. This is an open access article distributed under the terms of the Creative Commons Attribution License, which permits unrestricted use, distribution, and reproduction in any medium, provided the original author and source are credited.

Source of Support: Nil, Conflict of Interest: None declared. 